Egyptian Journal of Chemistry

http://ejchem.journals.ekb.eg/

\title{
Antimicrobial activity of new synthesized aza -beta lactam and tetrazole derivatives bearing imidazo[2,1-b]benzothiazole moiety
}

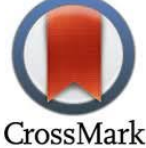

\author{
Kh. T. A. Al-Sultani*, N. Al-Lami \\ Department of Chemistry, College of sciences, Baghdad University, Baghdad, Iraq
}

\begin{abstract}
This research, included prepare of some new aza-beta lactam and 1,2,3, 4-tetrazole derivatives from 2aminobenzothiazole. The first step includes formation of imidazo[2,1-b]benzothiazoles (1) by the condensation of 2aminobenzathiazole and ethyl-2- chloro acetoacetate in acetone, then compound (1) reaction with hydrazine hydrate $80 \%$ to form hydrazone derivative (2). Schiff bases (3-6) were prepared from condensation of hydrazone(2) with various aromatic aldehyde with little drops of glacial acetic acid. Phenyl isocyanate and sodium azide were used for the cyclocyclization of new Schiff bases to form diazetidine (7-10) and tetrazole (11-14) derivatives. Moreover, Newly prepared derivatives were measured by Fourier-transform infrared and some of them by ${ }^{1} \mathrm{H} \&{ }^{13} \mathrm{C}-\mathrm{NMR}$. Furthermore, some new derivatives were evaluated as antibacterial.
\end{abstract}

Keywords: imidazo[2,1-b] Benzthiazole,Schiff base, Diazetidine, 1, 2, 3, 4-Tetrazole,antibacterial.

\section{$\underline{\text { Introduction }}$}

Imidazo benzothiazoles have been shown to be important heterocycles as a $[2,1-b]$ result of their pharmacological activities [1] such as Antiproliferative [2], anticancer [3], antifungal [4], anti-Alzheimer's disease [5], antibacterial [6], immunological activites [7] and antimicrobial [8]. These compounds have been prepared from various precursors by adopting different methods [9]. Schiff's bases is a branch of organic chemistry with a very high importance[10]. It was described for the first time by the German chemist Hugo Schiff in (1864). Schiff bases was made up by the condensation between primary amines and compounds containing carbonyl group, such as aldehyde, ketone in absolute alcohol and a few drops of glacial acetic acid [11,12]. Azetidinones are the carbonyl derivatives of azetidines, these are also known as 2-azetidinones or more commonly aza- $\beta$-1actams[13], Cyclocondensation of Schiff's bases with phenyl isocyanate yields 1,3-diazetidin -2-one[14]. Tetrazole consists of five member ring of four nitrogen and one carbon atom [15]. The $(2+3)$ cyclo addition method between nitriles and azides to be described common method to prepare the tetrazoles [16]. Tetrazole and their derivatives have different biological activities such as antifungal and antimicrobial [17]. Finally, this paper aims to synthesis and characterize some new azetidinones and tetrazole derivatives.

\section{Experimental section}

1. The FT-IR 8300 infrared spectrophotometer made up by SHIMADZU Company as a $\mathrm{KBr}$ disc was used as template in Science College, Baghdad University.

2. ${ }^{1} \mathrm{H}-\mathrm{NMR} \quad \&^{13} \mathrm{C}-\mathrm{NMR}$ spectral chemical shifts were measured on Bruker Mega Hertz by using DMSO- $\mathrm{d}_{6}$ as solvent.

3. Melting point (M.P.) was recorded by using Gallen Kamp melting point apparatus.

4. Antibacterial detection by Biology Department, Sciences College, University of Baghdad.

Synthesis of ethyl 2-methyl imidazo[2,1-b] benzthiazole-3-carboxylate [1] [18]

To solution of 2-chloro ethyl acetoacetate $(3.8 \mathrm{~mL}$, $0.028 \mathrm{~mol})$ under dry conditions, 2aminobenzothiazole $(2.635 \mathrm{~g}, 0.028 \mathrm{~mol})$ in acetone was added. To previous mixture, $\mathrm{K}_{2} \mathrm{CO}_{3}$ (3g) was added, and the resulting mixture was heated under reflux for $9 \mathrm{hrs}$. The end of reaction was checked by TLC. Acetone was evaporated by vacuum distillation and the residue treated with ethyl acetate and

*Corresponding author e-mail: khitam.t@sc.uobaghdad.edu.iq

Receive Date: 29 December 2020, Revise Date: 01 February 2021, Accept Date: 14 March 2021

DOI: 10.21608/EJCHEM.2021.55736.3175

C2021 National Information and Documentation Center (NIDOC) 
petroleum ether. The solid was filtered, dried then purified by recrystallization from ethanol to give compounds [1]. The Physical properties of compound [1] are listed in Table (1).

\section{Synthesis of 2-methylimidazo benzothiazole-3-carbohydrazide [2][19]}

Compound [1] $(0.01 \mathrm{~mol})$ was dissolved in $(15 \mathrm{~mL})$ abs. EtOH, and heated under reflux with hydrazine hydrate $80 \%(10 \mathrm{~mL})$ for $7 \mathrm{hrs}$. Then cooled, filtered and purified by recrystallization from chloroform to give compounds [2]. The Physical properties of compound [2] are listed in Table (2).
Synthesis of, $(E)$ - N'-(4- substituted benzylidene)2-methyl imidazo[2,1-b] benzthiazole-3carbohydrazide[3-6][11]

Series of Schiff bases compounds were prepared from the reaction of compound [3-6] $(0.003 \mathrm{~mol})$ in $(25 \mathrm{~mL})$ abs. EtOH, different aromatic aldehydes $(0.003 \mathrm{~mol})$ were added with little drops of glacial $\mathrm{AcOH}$. The resulting mixture was heated under refluxed for (6-7) hrs. Then, cold water was added, and the solid was obtained, filtered and purified by recrystallization from different appropriate solvents. The Physical properties of compound [3-6] are listed in Table

(3).

Table 1. The Physical properties of compound (1)

\begin{tabular}{|c|c|c|c|c|c|}
\hline \multirow{2}{*}{$\begin{array}{c}\text { Comp. } \\
\text { No. }\end{array}$} & \multicolumn{5}{|c|}{ Physical properties } \\
\hline & Structures & M.P. Co & Yield\% & Color & Res. \\
\hline 1 & & $\begin{array}{c}160 \\
\text { Decom. }\end{array}$ & 90 & $\begin{array}{l}\text { Light } \\
\text { brown }\end{array}$ & Ethanol \\
\hline
\end{tabular}

Table 2. The Physical properties of compound (2)

\begin{tabular}{|c|c|c|c|c|c|}
\hline \multirow{2}{*}{$\begin{array}{c}\text { Comp. } \\
\text { No. }\end{array}$} & \multicolumn{5}{|c|}{ Physical properties } \\
\hline & Structures & M.P. C & Yield\% & Colour & Res. \\
\hline & & & & & \\
\end{tabular}

Table 3. The Physical properties of compounds (3-6)

\begin{tabular}{|c|c|c|c|c|c|}
\hline \multirow{2}{*}{$\begin{array}{c}\text { Comp. } \\
\text { No. }\end{array}$} & \multicolumn{5}{|c|}{ Physical properties } \\
\hline & Structures & $\begin{array}{c}\text { M.P. } \\
\mathrm{C}^{\circ}\end{array}$ & $\begin{array}{c}\text { Yield } \\
\%\end{array}$ & Colour & Res. \\
\hline 3 & & $\begin{array}{c}126- \\
128\end{array}$ & 40 & $\begin{array}{c}\text { Dark } \\
\text { yellow }\end{array}$ & Acetone \\
\hline 4 & & $\begin{array}{c}186- \\
188\end{array}$ & 65 & $\begin{array}{c}\text { Pale } \\
\text { yellow }\end{array}$ & Methanol \\
\hline 5 & & $\begin{array}{c}175- \\
178\end{array}$ & 30 & Brown & Ethanol \\
\hline 6 & & $\begin{array}{l}210- \\
213\end{array}$ & 43 & $\begin{array}{c}\text { Off } \\
\text { white }\end{array}$ & Methanol \\
\hline
\end{tabular}


Synthesis of 2-methyl-N-(2-(4-substitutedphenyl)4-oxo-3-phenyl-1,3-diazetidin-1-yl) imidazo[2,1-b] benzthiazole-3-carboxamide[7-10][20].

A mixture of compounds [3-6] $(0.003 \mathrm{~mol})$ and phenylisocyanate $(0.003 \mathrm{~mol})$ in chloroform $(20 \mathrm{~mL})$ was refluxed for $6 \mathrm{hrs}$. Evaporation of solvent then the residue was treated with ethyl acetate: petroleum ether (1:1) as mixture. The solid was filtered, dried then purified by recrystallized from different appropriate solvents. The Physical properties of compound [7-10] are listed in Table (4).
Synthesis of N-(5-(4-substitutedphenyl)-1Htetrazol-1-yl)-2-methylimidazo[2,1-b]

benzthiazole-3-carboxamide[11-14][20]

A mixture of compounds [3-6] $(0.003 \mathrm{~mol})$ and $\mathrm{NaN}_{3}$ $(0.03 \mathrm{~g}, 0.003 \mathrm{~mol})$ in $(20 \mathrm{~mL})$ of $(\mathrm{THF})$ was added. The brevious mixture was heated under refluxe for (12-14) hrs. The solid was filtered, dried then purified by recrystallized from different appropriate solvents. The Physical properties of compound [11-14] are listed in Table (5).

Table 4. The Physical properties of compounds (7-10)

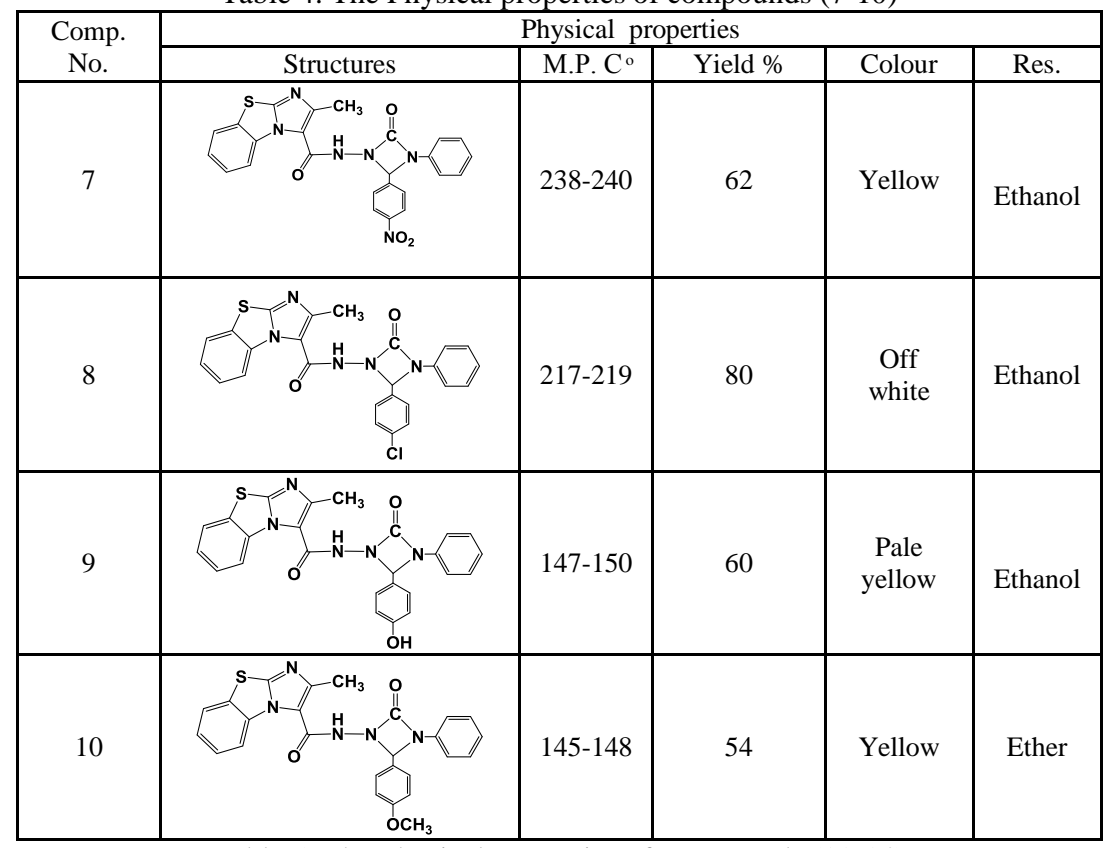

\begin{tabular}{|c|c|c|c|c|c|}
\hline \multirow{2}{*}{$\begin{array}{c}\text { Comp. } \\
\text { No. }\end{array}$} & \multicolumn{5}{|c|}{ Physical properties } \\
\hline & Structures & M.P. C ${ }^{\circ}$ & Yield $\%$ & Colour & Res. \\
\hline 11 & & $243-245$ & 44 & $\begin{array}{c}\text { Dark } \\
\text { yellow }\end{array}$ & Ethanol \\
\hline 12 & & $165-167$ & 90 & yellow & Ethanol \\
\hline 13 & & $\begin{array}{c}186 \\
\text { Decom. }\end{array}$ & 60 & Brown & Ethanol \\
\hline 14 & & $225-228$ & 75 & $\begin{array}{c}\text { Dark } \\
\text { brown }\end{array}$ & dioxane \\
\hline
\end{tabular}




\section{Results and Discussion}

Synthesis of new derivatives of diazetidine and 1,2,3,4-Tetrazole of 2-aminobenzothiazole found in scheme (1).

Reaction of 2-amino benzothiazole with ethyl-2chloroaceto acetate in dry acetone as solvent yield compound [1]. Ester test (hydroxamic acid) was gave positive indication of formation ester[21]. FT-IR spectrum of compound [1] exhibited of vanishing of $v(\mathrm{NH} 2)$ starching band at 3430 asym. $\mathrm{cm}^{-1}, 3184$ sym. $\mathrm{cm}^{-1}$ and revealing of feature bands at $2983 \mathrm{~cm}^{-1}$ belong $v(\mathrm{C}-\mathrm{H})$ aliphatic, 1724, 1267, and $1126 \mathrm{~cm}^{-1}$ which owing to of carbonyl,. Asym. and sym. v (C$\mathrm{O})$ of ester respectively [21]. Other bands exhibited at $1641 \mathrm{~cm}^{-1}$ and $1565 \mathrm{~cm}^{-1}$ owing to $v(\mathrm{C}=\mathrm{N})$ and $v(\mathrm{C}=\mathrm{C})$ aromatic [22]. The FTIR data of compound [1] are listed in Table (6). The ${ }^{1} \mathrm{H} \&{ }^{13} \mathrm{C}$ NMR signal date for compound [1] found in Tables (11) \&(12) respectively.

Hydrazide [2] was formed by reaction of ester [1] with hydrazine hydrate, This reaction occurred by nucleophilic attack of amino group on carbonyl group, after that elimination of ethanol. FT-IR spectrum of prepared compound [2] showed emergence of absorptions bands at $3350 \mathrm{~cm}^{-1}$ and at $3245 \mathrm{~cm}^{-1}$ sym of $\mathrm{NH}_{2}$ asym. and sym. Respectively, $3170 \mathrm{~cm}^{-1}$ of $\mathrm{NH}$ group, Also it showed shift in the $v \mathrm{C}=\mathrm{O}$ band from $1724 \mathrm{~cm}^{-1}$ of carbonyl of ester to $1649 \mathrm{~cm}^{-1}$ of amide. The FTIR data of compound [2] are listed in Table(7). The ${ }^{1} \mathrm{H}-\mathrm{NMR}$ signal date for compound [2] found in Tables (11).

Schiff's bases derivatives [3-6] were synthesized from the reaction of hydrazide [2] and a number of substituted aromatic aldehydes. The FTIR of compounds [2-7] includes the disappearance of $v(\mathrm{~N}-$ $\mathrm{H} 2$ ) absorption band and appearance of new bands at (1664-1610) $\mathrm{cm}^{-1}$ because of the formation of imine group $(\mathrm{C}=\mathrm{N})$. In addition to appearance of $v(\mathrm{CH})$ aromatic bands at (3066-3056) $\mathrm{cm}^{-1}, v(\mathrm{C}=\mathrm{O})$ amide absorption bands at 1697-1685 $\mathrm{cm}^{-1}$ and $v(\mathrm{C}=\mathrm{C})$ aromatic bands at (1512-1444) $\mathrm{cm}^{-1}$. The FTIR data of compound [3-6] are listed in Table (8). The ${ }^{1} \mathrm{H} \&$ ${ }^{13} \mathrm{C}$ NMR signal date for compound [6] found in Tables (11) \& (12) respectively.

Two different reagents were used in closing Schiff bases derivatives. The first method is when the Schiff bases reaction with phenyl isocyanate via [2+2] cycloaddition reaction producing Aza- $\beta$-lactam compounds of [7-10].

The FT-IR spectra showed the absence of the imine group $(\mathrm{CH}=\mathrm{N}-)$ absorption band at (1664-1610) $\mathrm{cm}^{-1}$ and the emergence new absorption band, of carbonyl group $(\mathrm{C}=\mathrm{O}$ aza- $\beta$-lactam $)$ at (1764-1712) $\mathrm{cm}-1$, these results gave a good evidence for the formation of the aza- $\beta$-lactam derivatives. The FTIR data of compound [7-10] are listed in Table (9). The ${ }^{1} \mathrm{H} \&$ ${ }^{13} \mathrm{C}$ NMR signal date for compound [7-10] found in Tables (11) \& (12) respectively.

Imine derivatives [3-6] reaction with $\mathrm{NaN}_{3}$ in second method to yield derivatives [11-14].

This reaction takes place according to $[3+2]$ cyclo addition of unsaturated systems to 1,3-dipoles to yield five-member ring [23].

FT-IR of compounds [11-14] showed peaks at (1539$15052)$ were owing $(\mathrm{N}=\mathrm{N})$ tetrazole ring. As well as, the FT-IR for these compounds emerge other bands at (1701-1666) $\mathrm{cm}^{-1}$, (1683-1604) $\mathrm{cm}^{-1}$ and (1610-1556) $\mathrm{cm}^{-1}$ due to carbonyl amide, $\mathrm{v}(\mathrm{C}=\mathrm{N})$ group and $v(\mathrm{C}=\mathrm{C})$ aromatic respectively[24]. The FTIR data of compound [11-14] are listed in Table (10). ${ }^{1} \mathrm{H}-\mathrm{NMR}$ and ${ }^{13} \mathrm{C}-\mathrm{NMR}$ spectrum of compound [11-14] listed in Table (11) and (12) respectively.

The antibacterial activity achieved by disk diffusion method[25]. The results of antibacterial activity are listed in Table (13).

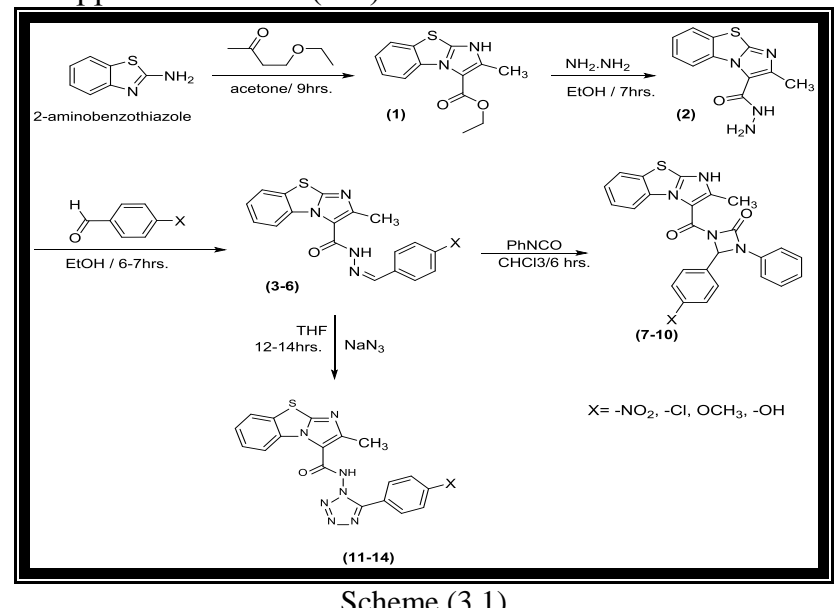

Scheme (3.1)

Egypt. J. Chem. 64, No. 6 (2021) 
Table 6. The FTIR spectral data of compound (1)

\begin{tabular}{|c|c|c|c|c|c|c|}
\hline \multirow{2}{*}{$\begin{array}{c}\text { Comp } \\
\text { No. }\end{array}$} & \multirow[b]{2}{*}{ Structures } & \multicolumn{5}{|c|}{ Major FTIR Absorption $\mathrm{Cm}^{-1}$} \\
\hline & & $\begin{array}{l}v \mathrm{C}-\mathrm{H} \\
\text { arom. } \\
\text { aliph. }\end{array}$ & $\begin{array}{c}v C=O \\
\text { ester }\end{array}$ & $v \mathrm{C}=\mathrm{N}$ & $\begin{array}{l}\nu \mathrm{C}=\mathrm{C} \\
\text { arom. }\end{array}$ & Others \\
\hline 1 & & $\begin{array}{l}3068 \\
2983\end{array}$ & 1724 & 1641 & 1565 & $\begin{array}{c}v(\mathrm{C}-\mathrm{O}) \\
\text { Ester } \\
1267 \\
1126\end{array}$ \\
\hline
\end{tabular}

Table 7. The FTIR spectral data of compound (2)

\begin{tabular}{|c|c|c|c|c|c|c|c|}
\hline \multirow[b]{2}{*}{$\begin{array}{c}\text { Comp. } \\
\text { No. }\end{array}$} & \multirow[b]{2}{*}{ Structures } & \multicolumn{6}{|c|}{ Major FTIR Absorption $\mathrm{Cm}^{-1}$} \\
\hline & & $\begin{array}{c}v\left(\mathrm{~N}-\mathrm{H}_{2}\right) \\
\text { asym. } \\
\text { Sym. }\end{array}$ & $v(\mathrm{~N}-\mathrm{H})$ & $\begin{array}{l}\mathrm{vC}-\mathrm{H} \\
\text { arom. } \\
\text { aliph. }\end{array}$ & $\begin{array}{l}v \mathrm{C}=\mathrm{O} \\
\text { Amid }\end{array}$ & $v \mathrm{C}=\mathrm{N}$ & $\begin{array}{l}v \mathrm{C}=\mathrm{C} \\
\text { arom. }\end{array}$ \\
\hline 2 & & $\begin{array}{l}3350 \\
3245\end{array}$ & 3170 & $\begin{array}{l}3066 \\
2964\end{array}$ & 1649 & 1577 & 1560 \\
\hline & $\mathrm{H}_{2} \mathrm{~N}^{\prime}$ & & & & & & \\
\hline
\end{tabular}

Table 8. The FTIR spectral data of compounds (3-6)

\begin{tabular}{|c|c|c|c|c|c|c|c|}
\hline \multirow[b]{2}{*}{$\begin{array}{c}\text { Comp. } \\
\text { No. }\end{array}$} & \multirow[b]{2}{*}{ Structures } & \multicolumn{6}{|c|}{ Major FTIR Absorption $\mathrm{Cm}^{-1}$} \\
\hline & & $v \mathrm{~N}-\mathrm{H}$ & $\begin{array}{l}\nu \mathrm{C}-\mathrm{H} \\
\text { arom. } \\
\text { aliph. }\end{array}$ & $\begin{array}{l}v \mathrm{C}=\mathrm{O} \\
\text { Amid }\end{array}$ & $\begin{array}{l}v \mathrm{C}=\mathrm{N} \\
\text { Imine } \\
\text { imidazo }\end{array}$ & $\begin{array}{l}v C=C \\
\text { arom. }\end{array}$ & Others \\
\hline 3 & & 3139 & $\begin{array}{l}3064 \\
2977\end{array}$ & 1697 & $\begin{array}{l}1623 \\
1596\end{array}$ & 1444 & $\begin{array}{c}v\left(\mathrm{NO}_{2}\right) \\
\text { Asym } \\
1521 \\
\text { sym1346 }\end{array}$ \\
\hline 4 & & 3473 & $\begin{array}{l}3066 \\
2941\end{array}$ & 1685 & $\begin{array}{l}1625 \\
1593\end{array}$ & 1490 & $\begin{array}{c}v \\
(\mathrm{C}-\mathrm{Cl}) \\
1091\end{array}$ \\
\hline 5 & & 3182 & $\begin{array}{l}3056 \\
2956\end{array}$ & 1695 & $\begin{array}{l}1664 \\
1604\end{array}$ & 1512 & $\begin{array}{c}v(\mathrm{C}-\mathrm{OH}) \\
3342\end{array}$ \\
\hline 6 & & 3434 & $\begin{array}{l}3060 \\
2997\end{array}$ & 1695 & $\begin{array}{l}1610 \\
1573\end{array}$ & 1502 & $\begin{array}{c}\nu \\
(\mathrm{C}-\mathrm{O}-\mathrm{C}) \\
1271,1157\end{array}$ \\
\hline
\end{tabular}


Table 9. The FTIR spectral data of compounds (7-10)

\begin{tabular}{|c|c|c|c|c|c|c|c|}
\hline \multirow[b]{2}{*}{$\begin{array}{c}\text { Comp } \\
\text { No. }\end{array}$} & \multirow[b]{2}{*}{ Structures } & \multicolumn{6}{|c|}{ Major FTIR Absorption $\mathrm{Cm}^{-1}$} \\
\hline & & $v \mathrm{~N}-\mathrm{H}$ & $\begin{array}{l}v \mathrm{C}-\mathrm{H} \\
\text { arom. } \\
\text { aliph }\end{array}$ & $\begin{array}{c}\nu \mathrm{C}=\mathrm{O} \\
\beta \text {-lactam } \\
\text { amid }\end{array}$ & $v \mathrm{C}=\mathrm{N}$ & $\begin{array}{l}v \mathrm{C}=\mathrm{C} \\
\text { arom. }\end{array}$ & Others \\
\hline 7 & & 3190 & $\begin{array}{l}3099 \\
2947\end{array}$ & $\begin{array}{l}1735 \\
1693\end{array}$ & 1591 & 1566 & $\begin{array}{c}v\left(\mathrm{NO}_{2}\right) \\
\text { asym1566 } \\
\text { sym1371 } \\
v(\mathrm{C}-\mathrm{N}) \\
1328\end{array}$ \\
\hline 8 & & 3164 & $\begin{array}{l}3083 \\
2991\end{array}$ & $\begin{array}{l}1764 \\
1699\end{array}$ & 1591 & 1527 & $\begin{array}{c}v(\mathrm{C}- \\
\mathrm{Cl}) 1081 \\
v(\mathrm{C}-\mathrm{N}) 1313\end{array}$ \\
\hline 9 & & 3290 & $\begin{array}{l}3058 \\
2987\end{array}$ & $\begin{array}{l}1712 \\
1649\end{array}$ & 1596 & 1552 & $\begin{array}{c}v(\mathrm{C}-\mathrm{OH}) \\
3328 \\
v(\mathrm{C}-\mathrm{N}) 1313\end{array}$ \\
\hline 10 & & 3211 & $\begin{array}{l}3090 \\
2971\end{array}$ & $\begin{array}{l}1712 \\
1652\end{array}$ & 1590 & 1554 & $\begin{array}{c}v(\mathrm{C}-\mathrm{O}-\mathrm{C}) \\
1240,1158 \\
v(\mathrm{C}-\mathrm{N}) \\
1340\end{array}$ \\
\hline
\end{tabular}

Table 10. The FTIR spectral data of compounds (11-14)

\begin{tabular}{|c|c|c|c|c|c|c|c|}
\hline \multirow[b]{2}{*}{$\begin{array}{c}\text { Comp. } \\
\text { No. }\end{array}$} & \multirow[b]{2}{*}{ Structures } & \multicolumn{6}{|c|}{ Major FTIR Absorption $\mathrm{Cm}^{-1}$} \\
\hline & & $v \mathrm{~N}-\mathrm{H}$ & $\begin{array}{l}\mathrm{vC}-\mathrm{H} \\
\text { arom. } \\
\text { aliph }\end{array}$ & $v \mathrm{C}=\mathrm{O}$ & $\nu \mathrm{C}=\mathrm{N}$ & $\begin{array}{l}v \mathrm{C}=\mathrm{C} \\
\text { arom. }\end{array}$ & Others \\
\hline 11 & & 3132 & $\begin{array}{l}3047 \\
2974\end{array}$ & 1681 & 1681 & 1595 & $\begin{array}{c}v \mathrm{~N}=\mathrm{N} \\
1519 \\
\mathrm{vNO}_{2} \\
\mathrm{Asym} \\
1519 \\
\text { sym1346 }\end{array}$ \\
\hline 12 & & 3450 & $\begin{array}{l}3099 \\
2987\end{array}$ & 1685 & 1623 & 1593 & $\begin{array}{c}v \mathrm{~N}=\mathrm{N} 1539 \\
v \mathrm{C}-\mathrm{Cl} \\
1089\end{array}$ \\
\hline 13 & & 3174 & $\begin{array}{l}3024 \\
2923\end{array}$ & 1666 & 1604 & 1556 & $\begin{array}{c}v(\mathrm{C}-\mathrm{OH}) \\
3303 \\
v(\mathrm{~N}=\mathrm{N}) 1514\end{array}$ \\
\hline 14 & & 3438 & $\begin{array}{l}3020 \\
2975\end{array}$ & 1701 & 1683 & 1610 & $\begin{array}{c}v \mathrm{~N}=\mathrm{N} 1502 \\
v \mathrm{C}-\mathrm{O}-\mathrm{C} \\
1271,1157\end{array}$ \\
\hline
\end{tabular}


Table 11. ${ }^{1} \mathrm{H}-\mathrm{NMR}$ spectral data $(\delta \mathrm{ppm})$ of some prepared compounds

\begin{tabular}{|c|c|c|}
\hline No. & Structures & ${ }^{1}$ HNMR Spectral data $\left({ }^{8} \mathrm{ppm}\right)$ \\
\hline 1 & & $\begin{array}{c}1.05\left(\mathrm{t}, 3 \mathrm{H},-\mathrm{CH}_{2}-\underline{\mathrm{CH}_{3}}\right) ; 2.20\left(\mathrm{~s}, 3 \mathrm{H}, \underline{\mathrm{CH}_{3}}\right) ; 4.11(\mathrm{q}, 2 \mathrm{H},-\mathrm{O}- \\
\left.\mathrm{C} \underline{\mathrm{H}}_{2}\right) ; 6.97-7.90(\mathrm{~m}, 4 \mathrm{H}, \mathrm{Ar}-\underline{\mathrm{H}})\end{array}$ \\
\hline 2 & & $\begin{array}{c}1.34\left(\mathrm{~s}, 3 \mathrm{H}, \mathrm{C} \underline{\mathrm{H}}_{3}\right) ; 4.70\left(\mathrm{~s}, 2 \mathrm{H}, \mathrm{N}_{2}\right) ; 6.97-7.84(\mathrm{~m}, 4 \mathrm{H}, \mathrm{Ar}- \\
\underline{\mathrm{H}}) ; 8.56(\mathrm{~s}, 1 \mathrm{H},-\mathrm{CO}-\mathrm{N}-\underline{\mathrm{H}}) 9.05(\mathrm{~s}, 1 \mathrm{H}, \mathrm{N}-\underline{\mathrm{H}})\end{array}$ \\
\hline 6 & & $\begin{array}{c}2.21\left(\mathrm{~s}, 3 \mathrm{H}, \mathrm{CH}_{3}\right) ; 3.81\left(\mathrm{~s}, 3 \mathrm{H}, \mathrm{OCC}_{3}\right) ; 6.98(\mathrm{~s}, 1 \mathrm{H}, \mathrm{N}=\mathrm{C}- \\
\underline{\mathrm{H}}) ; 7.00-7.96(\mathrm{~m}, 7 \mathrm{H}, \mathrm{Ar}-\underline{\mathrm{H}}) ; 8.82(\mathrm{~s}, 1 \mathrm{H}, \mathrm{CO}-\mathrm{N}-\underline{\mathrm{H}})\end{array}$ \\
\hline 9 & & $\begin{array}{c}1.23\left(\mathrm{~s}, 3 \mathrm{H}, \mathrm{CH}_{3}\right) ; 4.14\left(\mathrm{~s}, 1 \mathrm{H}, \mathrm{OCH}{ }_{3}\right) ; 6.62(\mathrm{~s}, 1 \mathrm{H}, \mathrm{CH} \text { aza- } \\
\beta-\mathrm{lactam}) ; 6.88-7.47(\mathrm{~m}, 14 \mathrm{H}, \mathrm{Ar}-\underline{\mathrm{H}}) ; \\
8.69(\mathrm{~s}, 1 \mathrm{H},-(\mathrm{CO})-\mathrm{NH}-) ; 9.59(\mathrm{~s}, 1 \mathrm{H}, \mathrm{OH})\end{array}$ \\
\hline 14 & & $\begin{array}{c}1.22\left(\mathrm{~s}, 3 \mathrm{H}, \mathrm{CH}_{3}\right) ; 3.60\left(\mathrm{~s}, 3 \mathrm{H}, \mathrm{OCH}_{3}\right) ; 6.62-7.91 \\
(\mathrm{~m}, 8 \mathrm{H}, \mathrm{Ar}-\underline{\mathrm{H}}) ; 8.82(\mathrm{~s}, 1 \mathrm{H}, \mathrm{N} \underline{\mathrm{H}})\end{array}$ \\
\hline
\end{tabular}

Table $12 .{ }^{13} \mathrm{C}$-NMR spectral data ( $\delta$ ppm) of some prepared compounds

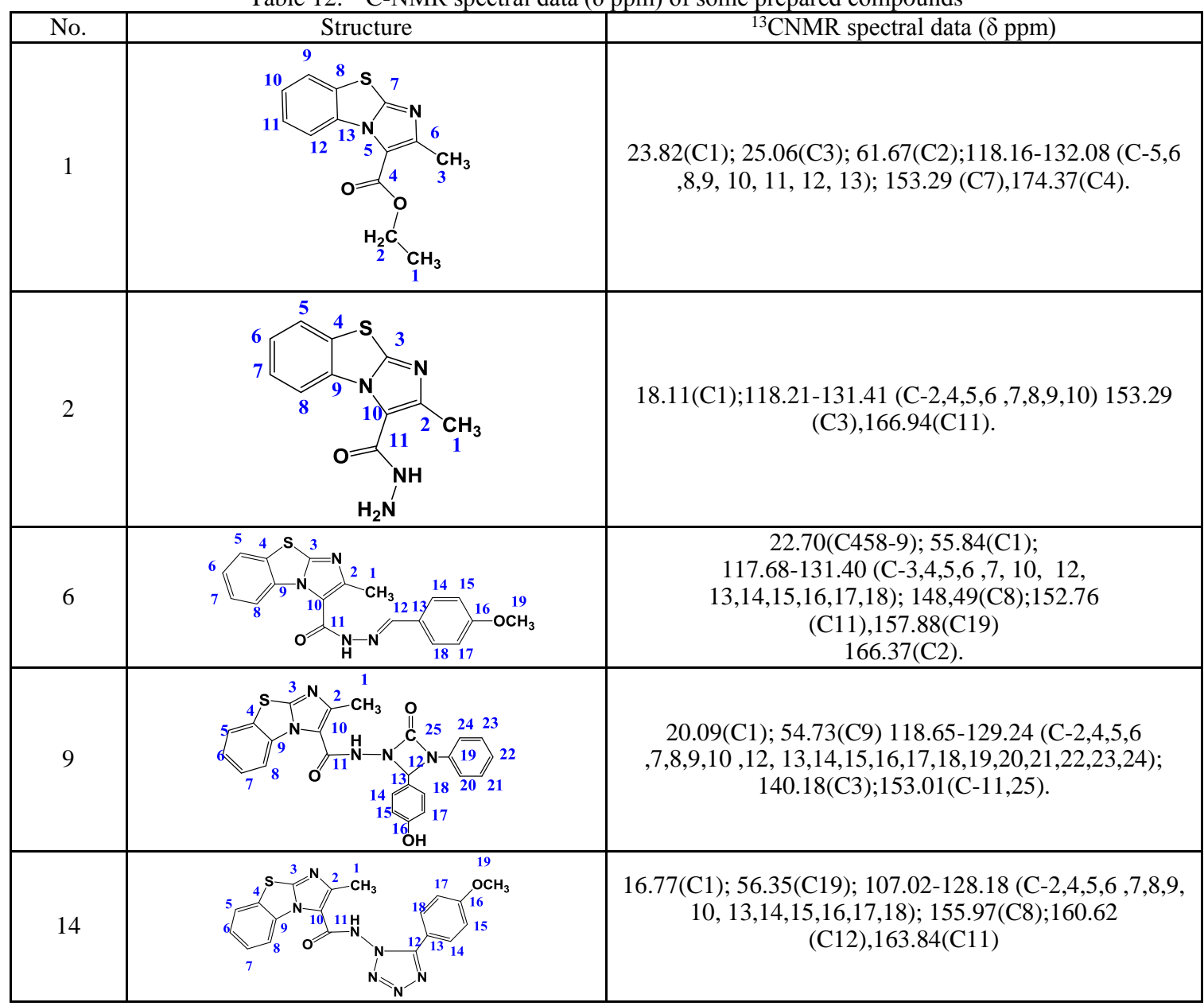


Table 13. Anti-bacterial activity for some prepared compounds

\begin{tabular}{|c|c|c|c|c|}
\hline $\begin{array}{c}\text { Comp. } \\
\text { No. }\end{array}$ & $\begin{array}{c}\text { Staphylococcus } \\
\text { aureus+ve }\end{array}$ & $\begin{array}{c}\text { Streptococcus } \\
+v e\end{array}$ & Klepsilla-ve & $\begin{array}{c}\text { Escherichia } \\
\text { coli-ve }\end{array}$ \\
\hline 4 & 11 & - & 26 & 32 \\
\hline 5 & 30 & 28 & 38 & 28 \\
\hline 7 & 13 & 15 & 11 & 13 \\
\hline 8 & 14 & 13 & - & 32 \\
\hline 12 & 26 & 26 & 30 & 32 \\
\hline 14 & 26 & 26 & 30 \\
\hline
\end{tabular}

\section{Conclusion}

Anew derivatives of imidazo(2,1-b)benzthiazole were evaluated against different types of strain cells of bacteria, and antibacterial tests showed promising results regarding inhibition activity of these types, where some of these derivatives exhibited strong activity, others showed moderate. Moreover, these results confirmed antimicrobial activities of imidazo/benzthiazole derivatives, which were reported in literatures. These derivatives were synthesised in five sequence steps, starting from 2amino benzthiazole and ended with introduced new five heterocycles of tetrazole and aza-beta lactam . Most of new derivatives were confirmed their structures precisely by FT-IR, ${ }^{1} \mathrm{H}-\mathrm{NMR},{ }^{13} \mathrm{C}-\mathrm{NMR}$ spectroscopy. These results encourage us to plan a new metholodgy of synthesis a new derivatives of imidazo/benzthiazole with study their pharmacoligal activities.

\section{$\underline{\text { References }}$}

[1] Sajjad M., Saeed E., Alireza M., Fereshteh T., Zohreh N. and Seyed J. Novel 99mTc-2-arylimidazo[2,1b]benzothiazole derivatives as SPECT imaging agents for amyloid-b plaques. Eur. J. Med. Chem., 175: 149161 (2019).

[2] Kumbhare R., Kumar K. V., Ramaiah M. J., Dadmal T., Pushpavalli S., Mukhopadhyay D., Divya B., Devi T., Kosurkar U. and Pal-Bhadra M. Synthesis and biological evaluation of novel Mannich bases of 2arylimidazo [2, 1-b] benzothiazoles as potential anticancer agents. Eur. J. Med. Chem., 46(9): 4258-4266 (2011).

[3] Ali I., Fozia B., Syeda A., Amjad I., Sameh M., Alessio N., Siham A. and Claudiu T. Benzothiazole derivatives as anticancer agents. J. Enzyme Inhibit. Med. Chem., 35(1): 265-279 (2020).

[4] Koudad M., El Hamouti C., Elaatiaoui A., Dadou S., Oussaid F., Pilet G., Benchat N. and Allali M. Synthesis, crystal structure, antimicrobial activity and docking studies of new imidazothiazole derivatives. $J$. Iran. Chem. Soc., 17: 297- 306 (2020).

[5] Alagille D., DaCosta H., Baldwin R. and Tamagnan G. 2-Arylimidazo [2, 1-b] benzothiazoles: A new family of amyloid binding agents with potential for PET and SPECT imaging of Alzheimer's brain. Bio. Med. Chem. Lett., 21(10): 2966-2968 (2011).

[6] Singh Y., Kaur B., Kaur A., Gupta V. and Gupta M. Synthesis, spectral studies and biological activity of 2, 3-disubstituted imidazo [2, 1-b] benzothiazole derivatives. Indian J. Pharm., 6(1): 1-8 (2018).

[7] Mase T., Arima T., Tomioka K., Yamada T. and Murase K. Imidazo [2, 1-b] benzothiazoles. 2. New immunosuppressive agents. J. Med. Chem., 29(3): 386-394 (1986).

[8] Swetha K., Zhen L., Vijaya K., Rammohan R., Balaraju T., Vijai K. and Tangadanchua C. Azoalkyl ether imidazo[2,1- b ]benzothiazoles as potentially antimicrobial agents with novel structural skeleton. Bio. Med. Chem. Lett., 28(14): 2426-2431 (2018).

[9] Ali I., Fozia B., Syeda A., Amjad I., Sameh M., Alessio N., Siham A. and Claudiu T. Benzothiazole derivatives as anticancer agents. $J$. Enzyme Inhibit. Med. Chem., 35(1): 265-279 (2020).

[10]Khitam T. A. Preparation Some New Heterocyclic Compounds Derived from Schiff Bases and Evaluation its Biological Activity. Int. J. Sci. Res., 6 (5):1567-1573 (2017).

[11]Khitam T. A. A., Suaad M. H. A. and Oday H. R. A. Synthesis, Identification and Evaluation Biological Activity for Some New Triazole, Triazoline and Tetrazoline Derivatives From 2Mercapto-3-phenyl-4(3H)Quinazolinone. Iraqi J. Sci., 57(1B): 295-308 (2016).

[12]Milan C., Maja M., Bojan S., Elizabeta H. and Valentina R. Synthesis and Antioxidant Activity of Some New Coumarinyl-1,3-Thiazolidine-4nes. Molecules, 15(10): 6795-6809 (2010).

[13]Suschitzky H. and Scriven E. Progress in Heterocyclic Chemistry. Pergamon Press. Oxford., 6: 206 (1994).

[14]Naeemah A. and Khawla J. S. Synthesis and Biological Activity Evaluation of New Imidazo and Bis Imidazo (1, 2-A) Pyridine Derivatives. $J$. Global Pharma. Technol., 10(11): 603-611 (2018). 
[15]Jalal H. M. Biological activities importance of Tetrazole derivatives. Eur. Acad. Res., 3(12): 12796-12804 (2016).

[16]Myznikov L. V., Vorona S. V., Artamonova T. V. and Zevatskii E. Y. Mechanism of the ZincCatalyzed Addition of Azide Ion to Unsaturated Compounds: Synthesis of 5-Substituted $1 \mathrm{H}$ Tetrazoles from Nitriles and of 1 -Substituted $1 H$ Tetrazole-5-thiols from Isothiocyanates. Russian J. Gen. Chem., 87(4): 731-738 (2017).

[17]Shobanbabu B., Narsimha R. P., Meenakshisundaram B., Sudhakiranmayi K., Eloisi C., Monica L. G., Ramesh B. and Peter A. C. A novel tetrazole analogue of resveratrol is a potent anticancer agent. Bio. Med. Chem., Lett., 29: 172-178 (2019).

[18]Valentina P., Ilango K. and Anita P. K. Synthesis, docking studies and biological evaluation of some newly substituted-5-(2 methyl imidazo [1,2-a] pyridin-3 -yl) $-2,5$ dihydro -1,3,4 -thiadiazol-2-amines. Int. J. Pharm Pharm. Sci., 5:3872-876 (2013).

[19]Mohammed G. A. A. and Suaad M. H. A. Synthesis, Characterization and Evaluation Antimicrobial Activity of Some New substituted
2-Mercapto-3-Phenyl-4(3H)-Quinazolinone. Iraqi J. Sci., 55(2B): 582-593 (2014).

[20]Mohammed R. A., Suaad M. H. A. and Ayad K. K. Synthesis, Evaluation Antimicrobial Activity of Some New $N$-substituted Naphthalimides Containing Different Heterocyclic Rings. Iraqi J. Sci., 54(4): 761-774 (2013).

[21]Ralph L. S., Christine K. F. H., Terence C. M., David Y. C. and Reynolo C. F. The systematic Identification of Organic Compounds. $8^{\text {th }}$ ed., John Wiley and Sons, New York (1980).

[22]Koj N. Infrared abstraction Spectroscopy. $1^{\text {st }}$ ed., Nankodo Company Limited, Tokyo (1962).

[23]Theophil E. and Siegfried H. The chemistry of heterocycles. $2^{\text {nd }}$ ed. Wiley-VCH GmbH \& Co. KGaA, Germany (2003).

[24]Silverstein R. M., Webster F. X. and Kiemle D. J. Spectrometric Identification of Organic Compounds. John Wiley \& Sons (2014).

[25]Anesini C. and Perez C. Screening of plants used in argentic folk medicine for antibacterial activity. J. Ethnropharm., 39(2): 35-47 (1993).

\title{
الفعالية المضادة للمايكروبات لمشتقات جديدة من ازو - بيتالاكتام وتترازول محضرة من جزيئة

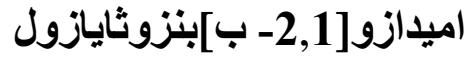

\author{
ختام طارق احمد، نعيمة جبار عويد \\ اقفم الكبياء، كلية العلوم، جامعة بغد/د، بغد/د، العر/ق
}

الخلاصة

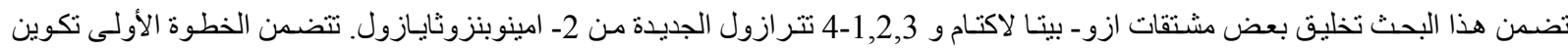

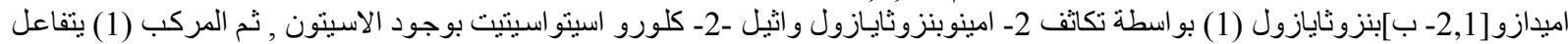

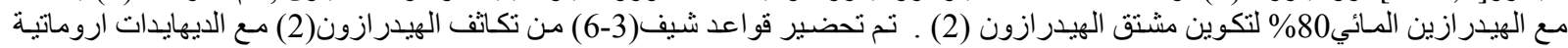

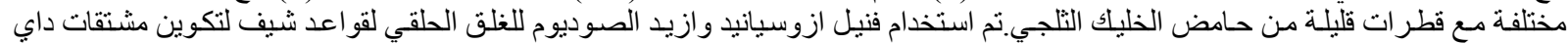

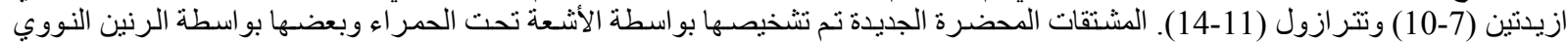

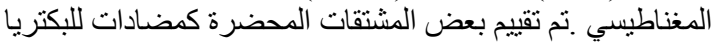

